\title{
DISEÑO DE UN BIORREACTOR PARA LA PRODUCCIÓN INDUSTRIAL DE VINAGRE DE MANZANA
}

\section{DESIGN OF A BIORREACTOR FOR THE INDUSTRIAL PRODUCTION OF VINAGRE FOR APPLE}

\author{
Brito-Terán Jorge ${ }^{1 *}$; García Valery²; Intriago-Intriago Lorena ${ }^{3}$; Vélez Carla ${ }^{4}$ Zambrano \\ Génesis $^{5}$; Rosero Ernesto ${ }^{6}$ \\ 1,2,3,4,5Estudiantes de Ingeniería de los Bioprocesos, Programa de Ingeniería Química \\ de la Facultad de Ciencias Matemáticas, Físicas y Químicas, Universidad Técnica de \\ Manabí. Portoviejo, Ecuador. \\ ${ }^{6}$ Docente de Ingeniería Química de la Facultad de Ciencias Matemáticas, Físicas y \\ Químicas, Universidad Técnica de Manabí. Portoviejo, Ecuador.
}

*Correo: jbrito8475@utm.edu.ec

\begin{abstract}
Resumen
El ácido acético tiene una gran importancia ya que se utiliza como aromatizante en ensaladas y otros alimentos, y debido a su acidez, también se emplea en la elaboración de encurtidos, por esta razón la presente investigación tiene como objetivo el diseño de un biorreactor para la producción industrial de vinagre de manzana, teniendo en cuenta las diversas variables que se puedan presentar, lo cual garantiza un mayor rendimiento de producción. El método empleado para el proceso de fermentación acética es el método Orleans, para poder así determinar los coeficientes de la ecuación de cultivo celular, los coeficientes de rendimientos del proceso, balance de materia y energía del proceso, y de esta forma desarrollar los cálculos para un diseño del biorreactor. Los resultados obtenidos fueron un YP/Smax $=1,95$ y un $\mathrm{YB} / \mathrm{Smax}=5,342$ con un diseño de biorreactor con $0,14 \mathrm{~m}^{3}$ y con una altura de $1 \mathrm{~m}$, con lo que se pudo llegar a concluir que las bacterias transformaron el $60 \%$ del sustrato alcohólico, lo cual es referente para generar una propuesta del diseño de un biorreactor a escala industrial.
\end{abstract}

Palabras clave: biorreactor, vinagre, fermentación, ácido acético.

\begin{abstract}
Acetic acid is of great importance since it is used as a flavoring in salads and other foods, and due to its acidity, it is also used in the preparation of pickles, for this reason the present research aims to design a bioreactor for Industrial production of apple cider vinegar, taking into account the various variables that may occur, which guarantees a higher production yield. The method used for the acetic fermentation process is the Orleans method, in order to determine the coefficients of the cell culture equation, the coefficients of yields of the process, balance of matter and energy of the process, in order to develop the calculations for a design of the bioreactor. The results obtained were a YP / Smax $=1.95$ and a YB / Smax $=5.342$ with a bioreactor design with $0.14 \mathrm{~m}^{3}$ and a height of $1 \mathrm{~m}$, so it was concluded that bacteria transformed $60 \%$ of the substrate alcoholic, which is a reference for the design of an industrial scale bioreactor.
\end{abstract}

Keywords: bioreactor, vinegar, fermentation, acetic acid

Información del manuscrito:

Fecha de recepción: 07 de mayo de 2019

Fecha de aceptación: 21 de junio de 2019

Fecha de publicación: 10 de julio de 2019 


\section{Introducción}

El hombre ha utilizado desde tiempos remotos el vinagre en la cocina, bien por su sabor ácido o como conservante de alimentos. Es un excelente condimento que contiene cerca de un $5 \%$ de ácido acético. Sin lugar a dudas el Vinagre de manzana es el más reconocido, sin embargo, cualquier fruta es buena para elaborar este producto, siendo posible obtener una gran variedad de sabores como: vinagres de higos, piña, plátano, mango, fresa, mandarina, naranja, ciruela, durazno, así como también de miel de abeja, cerveza, inclusive se pueden aromatizar con hierbas 0 especias (romero, menta, estragón, ajo, cebollines, entre otros) (Aleixandre et al., 2003).

Es de conocimiento para muchos que la nueva revolución industrial está presente en el mundo, y nadie desea quedarse atrás u obsoleto por lo que el desarrollo de nuevas alterativas que permitan la optimización de los procesos se consideran una solución primordial y que garantiza estar en la vanguardia industrial a nivel mundial (Heikefelt, 2011).
En la actualidad las personas se encuentran ante la próxima revolución industrial llamada Biotecnología, todo esto basado en la capacidad de los microorganismos de producir diversos tipos de productos enfocados a la industria farmacéutica, alimenticia y diversos compuestos químicos, la cual aplicando diversos mecanismos puede ser orientada y controlada con fines específicos (Shishehbor et al., 2008).

Un biorreactor o fermentador, es una pieza clave y central de estos procesos biotecnológicos, ya que este proporciona un ambiente controlado que permite el crecimiento eficaz de las células y la formación de un producto; todo esto teniendo niveles óptimos de temperatura, ph, sustrato, oxígeno; para así convertir la materia prima en productos de interés específicos. Es por ello que el presente artículo tiene como objetivo el diseño de un biorreactor para la producción industrial de vinagre de manzana, teniendo en cuenta las diversas variables que se puedan presentar, lo cual garantice un mayor rendimiento de producción (Johnston et al., 2010). Los procesos 
biotecnológicos en el país presentan un gran potencial a nivel económico a corto y largo plazo; es por ello que los diversos sectores industriales presentes se enfocan en buscar diversas alternativas que potencien sus producciones, permitiendo así un avance no solo para este sector sino para diversos sectores económicos y fomentando la competencia con el mercado internacional (Jimenez-Hornero et al., 2009).

\section{Materiales y métodos}

El proceso para la elaboración de vinagre de manzana se realiza a partir del vino de manzana, con un acondicionamiento del sustrato alcohólico, inoculación y acetificación. Cada etapa en la que se tomaron muestras para las diferentes determinaciones analíticas se identifica con la sigla (TM). En el caso de las muestras tomadas durante el proceso de acetificación, se expresó como g/L de ácido acético (se asume que toda la acidez del medio se debe a este ácido).

\subsection{Recepción de la materia prima}

Para este estudio se empleó un vino artesanal de manzana proveniente del cantón Sucre, se seleccionaron los vinos artesanales claros, limpios y libres de materias extrañas.

\subsection{Obtención del inóculo}

El microorganismo utilizado para la fermentación acética fue Acetobacter Aceti obtenido a partir de la "madre de vinagre" de un vinagre de banano artesanal proveniente del cantón Rocafuerte. La madre está compuesta tanto de bacterias acéticas como de levaduras, que trabajan juntas (Alawad et al., 2018).

\subsection{Biorreactor a escala de laboratorio}

Se emplearon botellas de vidrio de $500 \mathrm{ml}$ de capacidad, con $7 \mathrm{~cm}$ de diámetro, selladas con gasa para evitar la contaminación del medio fermentativo.

\subsection{Descripción del proceso de fermentación}

Para la ejecución de la experimentación se prepararon en cinco recipientes de vidrio previamente esterilizados, un medio de $100 \mathrm{ml}$ que contenía alcohol al $15 \% \mathrm{v} / \mathrm{v}$ (diluido con agua), $0,15 \mathrm{~g}$ de sulfato de amonio como fuente de nitrógeno y $0,01 \mathrm{~g}$ de sorbato de 
potasio para inhibir el crecimiento de levaduras, se inoculó con el 10\% p/p de "Madre del vinagre" (Mycoderma aceti) (Ferreyra et al., 2014), es decir, $10 \mathrm{~g}$ de Mycoderma aceti (2,5 gramos en peso seco). Se llevó a cabo la fermentación por un período de 5 días, tapando los recipientes con gasa para el ingreso de aire del medio exterior y evitando el ingreso de otras partículas. La fermentación fue realizada en condiciones ambientales de temperatura y presión.

\subsection{Medición de las variables experimentales}

- Grados de alcohol.

Se realizó la determinación previa del porcentaje de alcohol v/v al vino inicial para conocer el contenido de sustrato presente, en caso que este sea mayor al $15 \%$ v/v se necesitará de una dilución, debido a que las bacterias acéticas no toleran altas concentraciones de alcohol (Ferreyra et al., 2014).

Además, cada 24 horas se realizó la determinación de este parámetro, para obtener el consumo de sustrato, indispensable para los cálculos posteriores, utilizando un refractómetro para alcohol de vinos
80ATC V/V 0-80\%, que mide la concentración de alcohol en vinos o solución acuosa expresado en porcentaje $\mathrm{v} / \mathrm{v}$.

- Biomasa.

La fórmula para la biomasa de Acetobacter aceti utilizada para los cálculos es $\mathrm{CH}_{1,8} \mathrm{O}_{0,5} \mathrm{~N}_{0,2}$ (Hurtado et al., s.f.). Para este estudio se consideró el valor de peso seco de la madre de vinagre, mediante el secado en estufa a $80^{\circ} \mathrm{C}$ (de Ory et al., 2002), asumiendo que todo el peso corresponde a Acetobacter aceti.

- Ácido acético.

Para determinar la acidez del vinagre se realiza una valoración con $\mathrm{NaOH}$ al $0,09 \mathrm{~N}$. Se transfieren $10 \mathrm{ml}$ de vinagre y se procede a realizar la titulación usando como indicador la fenolftaleína hasta alcanzar el punto de equilibrio.

- Temperatura y $\mathrm{pH}$.

Se realizó un monitoreo diario de ambos parámetros para evaluar las condiciones del medio, se utilizó un termómetro de mercurio ASTM 12F y tiras de $\mathrm{pH}$. 
- Caudal de aire.

La eficiencia en la aireación de un sistema fermentativo depende de la solubilidad del oxígeno, la velocidad de difusión en los medios de cultivo, la demanda de oxígeno del cultivo y la capacidad del biorreactor de satisfacer dicha demanda en la población microbiana (Soler \& Buitrago, 2010), para este ensayo se determinó el flujo de aire a través de la difusión de este en el medio fermentativo, se empleó la ecuación de difusión propuesta en Geankopolis (1999). Tal como se presenta en este texto, el flujo molar de aire se encuentra en función del coeficiente de difusión, constantes de gases, temperatura del ensayo y nivel del medio fermentativo. Por su parte, el coeficiente de difusión del aire-ácido acético se determinó mediante la fórmula que considera la temperatura a la que se realiza el ensayo, peso molecular del aire y ácido acético, presión a la que se realiza el ensayo, volumen atómico de difusión de aire y ácido acético.

Una vez que se conoce el valor del flujo del aire que se difunde, por fracción másica se determina el flujo de oxígeno y nitrógeno en $\mathrm{Kg} / \mathrm{m}^{2} \mathrm{~s}$, se realiza una relación en base al área del biorreactor y el tiempo de fermentación. Se considera la densidad del aire a la temperatura promedio $\left(26^{\circ} \mathrm{C}\right): 1,175 \mathrm{~kg} / \mathrm{m} 3$ (Geankopolis, 1999) y mediante los porcentajes másicos de oxígeno y nitrógeno que son $23,7 \%$ y $79,3 \%$, respectivamente (Doran, 2014), es posible conocer el flujo de estos componentes durante el proceso de acetificación.

\subsection{Diseño del biorreactor a escala industrial}

A través del balance de masa del proceso, es posible dar una guía referente al diseño de un biorreactor para la producción de ácido acético tomando como referencia la cantidad actual de producción, la misma que para el año 2015 fue de 196 toneladas (Teneda-Llerena \& MillaToro, 2018) con un porcentaje de ácido acético del $6 \%$ de acuerdo a la normativa NTE INEN 2296:2013. El diseño del biorreactor es en base a sus dimensiones como son el diámetro y altura del fermentador junto con la implementación de un impelente de Turbina Rushton, se determinó el diámetro del impelente, ancho de rodete y espacio libre de la parte inferior. 


\section{Resultados y discusión}

\subsection{Contenido de alcohol}

En la Tabla 1 se presenta la disminución de los grados de alcohol durante los 5 días de fermentación. En este proceso, el $60 \%$ del alcohol contenido en el vino se convirtió en ácido acético y anhídrido carbónico, eso se realizó de forma gradual con la disminución de $1 \% \mathrm{v} / \mathrm{v}$ cada 24 horas durante 5 días, en base a estos datos es posible establecer la gráfica de la cinética de reducción del alcohol etílico.

Tabla 1.

Medición de los grados de alcohol

\begin{tabular}{|c|c|c|}
\hline Día & \% v/v Alcohol & Sustrato $(\mathrm{g})$ \\
\hline 0 & 15 & 11,83 \\
\hline 1 & 13 & 10,25 \\
\hline 2 & 12 & 9,46 \\
\hline 3 & 11 & 8,67 \\
\hline 4 & 10 & 7,89 \\
\hline 5 & 9 & 7,10 \\
\hline
\end{tabular}

\subsection{Producción de biomasa}

La Tabla 2 muestra la producción de biomasa medida bajo condiciones experimentales. Durante el período de fermentación se observó un velo de color blanquecino sobre la superficie del medio, de características similares al inóculo, es decir un material gelatinoso, al transcurso de los días fue más visible, por lo cual se evidencia la generación de biomasa por reproducción celular que aumenta con respecto al tiempo.

Tabla 2.

Producción de biomasa

\begin{tabular}{|c|c|}
\hline Día & Peso seco $(\mathrm{g})$ \\
\hline 0 & 2,5 \\
\hline 1 & 3 \\
\hline 2 & 4,2 \\
\hline 3 & 5 \\
\hline 4 & 5,62 \\
\hline 5 & 6,23 \\
\hline
\end{tabular}

\subsection{Producción de ácido acético}

En la Tabla 3 se puede observar que la producción de ácido acético aumentó conforme se desarrolló el proceso. Se arrancó la corrida experimental con 0,072 gramos de ácido acético y se finalizó al quinto día con 1,35 gramos.

\section{Tabla 3.}

Producción de ácido acético

\begin{tabular}{|c|c|}
\hline Día & Ácido acético $(\mathrm{g})$ \\
\hline 0 & 0,072 \\
\hline 1 & 0,209 \\
\hline 2 & 0,792 \\
\hline 3 & 0,936 \\
\hline 4 & 1,188 \\
\hline 5 & 1,35 \\
\hline
\end{tabular}

\subsection{Control de temperatura y $\mathrm{pH}$}

En la Tabla 4 se registra el monitoreo de la temperatura y $\mathrm{pH}$ del medio, esto con la finalidad de mantener el medio óptimo para el crecimiento y reproducción del microorganismo. 
Tabla 4.

Mediciones de temperatura y $\mathrm{pH}$

\begin{tabular}{|c|c|c|}
\hline Día & Temperatura $\left({ }^{\circ} \mathrm{C}\right)$ & $\mathrm{pH}$ \\
\hline 0 & 26 & 6 \\
\hline 1 & 26 & 6 \\
\hline 2 & 27 & 6 \\
\hline 3 & 27 & 5,5 \\
\hline 4 & 26 & 5 \\
\hline 5 & 26 & 5 \\
\hline
\end{tabular}

El pH se mantuvo fluctuando entre valores de 5 a 6 , siendo un medio selectivo para las bacterias del género Acetobacter. El pH óptimo para el desarrollo del microorganismo debe ser un medio ácido (de Ory et al., 2002). Esto pudo resultar beneficioso para el proceso ya que los vinagres obtenidos de materias primas naturales muestran cierta inestabilidad con respecto a la solubilidad de las sustancias previamente disueltas. Cuanto menos cambian los valores de $\mathrm{pH}$ durante la fermentación, mayor será la estabilidad (Solieri, 2009).

\subsection{Caudal de aire que se difunde en el medio fermentativo}

El biorreactor a escala de laboratorio tiene una altura de 0,035 metros, con un diámetro de 0,07 metros y un área de fermentación de 0,0115 $\mathrm{m}^{2}$, considerando al reactor como un cilindro.

En la Tabla 5 se tienen los datos de los volúmenes atómicos de difusión para el aire y ácido acético, para el cálculo de la difusividad y flujo molar de aire durante las 120 horas de fermentación, se tomó un valor promedio de temperatura ambiente de $26^{\circ} \mathrm{C}$, considerando 1 atmósfera de presión, además de que el valor de la constante de gases ideales $R$ fue $8314 \mathrm{~J} / \mathrm{kmol}^{\star} \mathrm{K}$. Se obtienen 456,48 gramos de oxígeno y 1502,58 gramos de nitrógeno que ingresaron al medio durante el período de fermentación.

Tabla 5.

Condiciones para el flujo de aire del proceso

\begin{tabular}{|c|c|}
\hline$v_{A}{ }^{*}$ & 20,1 \\
\hline$v_{B}{ }^{*}$ & 51,88 \\
\hline$D_{A B}$ & $1,1703 \times 10^{-5} \frac{\mathrm{m}^{2}}{\mathrm{~s}}$ \\
\hline$N_{A}$ & $0,067928 \mathrm{Kgmol}$ \\
\hline
\end{tabular}

\subsection{Coeficientes estequiométricos}

y rendimientos máximos de productos

La Tabla 6 presenta una comparación de los rendimientos obtenidos experimentalmente con los máximos termodinámicos, a partir del rendimiento de la biomasa obtenido en la fermentación. En los experimentos de laboratorio, la producción de ácido acético empezó inmediatamente, tal como se muestra en la Tabla 3 , sin ninguna 
fase de latencia mostrada durante los 5 días que duró el proceso. Sin embargo, en comparación con el rendimiento de producto $\left(\mathrm{Y}_{\mathrm{P} / \mathrm{S}}\right)$ la reacción favoreció a la producción de biomasa y no a la formación esperada de ácido acético, según como se muestra en la tabla 6 , esto debido a que son varios los factores que influyen en el rendimiento de la biomasa, incluido la composición del medio, la naturaleza de las fuentes de carbono y nitrógeno, $\mathrm{pH}$ y temperatura. Además, el rendimiento de la biomasa $\left(\mathrm{Y}_{\mathrm{B} / \mathrm{S}}\right)$ es mayor en cultivos aerobios que en cultivos anaerobios, la elección del aceptor de electrones (p. ej., oxígeno molecular, nitrato o sulfato) también puede tener un efecto significativo (Doran, 2013).

\section{Tabla 5.}

Rendimientos experimentales y máximos del proceso de fermentación acética

\begin{tabular}{|c|c|}
\hline \multirow{4}{*}{$\begin{array}{c}\text { Ácido } \\
\text { acético }\end{array}$} & $\begin{array}{c}\text { Rendimiento experimental } \\
0,270 \mathrm{~g} / \mathrm{g}\end{array}$ \\
\cline { 2 - 2 } & $\begin{array}{c}\text { Rendimiento máximo } \\
\text { termodinámico }\end{array}$ \\
& $\boldsymbol{y}_{\boldsymbol{P} / \mathrm{s} \text { mix }}=\mathbf{1 , 9 5} \frac{\mathrm{g} \text { A.Acético }}{\mathrm{g} \text { Etanol }}$ \\
\hline \multirow{4}{*}{ Biomasa } & $\begin{array}{c}\text { Rendimiento experimental } \\
0,788 \mathrm{~g} / \mathrm{g}\end{array}$ \\
\cline { 2 - 2 } & $\begin{array}{c}\text { Rendimiento máximo } \\
\text { termodinámico }\end{array}$ \\
& $\boldsymbol{y}_{\boldsymbol{B} / \mathbf{s} \text { mix }}=\mathbf{5 , 3 4 2} \frac{\mathrm{g} \text { Etanol }}{\mathrm{g} \text { Biomasa }}$ \\
\hline
\end{tabular}

A partir de los rendimientos obtenidos, se puede generar un balance de materiales del proceso de producción de ácido acético.

Tabla 6.

Balance de masa del proceso fermentativo anaerobio

\begin{tabular}{|c|c|c|c|c|c|}
\hline Compuesto & Entrada (mol) & Genera (mol) & Consume (mol) & Sale (mol) & Sale (gramos) \\
\hline $\mathrm{C}_{2} \mathrm{H}_{6} \mathrm{O}$ & 0,2571 & 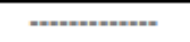 & 0,1028 & 0,1543 & 7,10 \\
\hline $\mathrm{O}_{2}$ & 14,265 & (................. & 0,1066 & 14,1584 & 453,069 \\
\hline $\mathrm{N}_{2}$ & 53,664 & ..... & $\cdots$ & 53,664 & 1502,58 \\
\hline $\mathrm{NH}_{3}$ & $8,82 \times 10^{-3}+0,0302$ & (................. & $8,82 \times 10^{-3}+0,0302$ & 0 & 0 \\
\hline Biomasa & 0,1016 & 0,1515 & (n................ & 0,2531 & 6,23 \\
\hline $\mathrm{CO}_{2}$ & 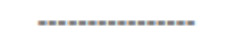 & 0,01151 & n................. & 0,01151 & 0,506 \\
\hline $\mathrm{H}_{2} \mathrm{O}$ & $4,722+0,416$ & 0,1748 & (.................. & 5,3128 & 95,6 \\
\hline $\mathrm{C}_{2} \mathrm{H}_{4} \mathrm{O}_{2}$ & $1,2 \times 10^{-3}$ & 0,0212 & (................. & 0,0224 & 1,34 \\
\hline TOTAL & 73,466 & & & 73,576 & \\
\hline
\end{tabular}

3.7. Diseño del biorreactor a escala industrial
Debido a que el proceso de producción de ácido acético a partir 
de vino de manzana a escala de laboratorio, tuvo un rendimiento global bajo (13,85\%), para optimizar el proceso, se diseñó un reactor con características más técnicas ya que según Drysdale \& Fleet (1988), cuando la concentración de $\mathrm{O}_{2}$ es demasiado baja, el proceso se ralentiza y se favorecen ciertas reacciones secundarias, como la formación de acetato de etilo por esterificación de etanol y ácido acético. Por lo tanto, es necesario lograr una buena transferencia de oxígeno en el fermentador, generalmente por medio de una aireación intensiva y una mezcla adecuada de aire y fases líquidas (Drysdale \& Fleet, 1988).

Tabla 7.

Cantidades de producción

\begin{tabular}{|l|l|l|}
\hline \multicolumn{1}{|c|}{ Producción de: } & \multicolumn{2}{c|}{ Cantidades } \\
\hline Manzanas & 196 ton/año & $96 \mathrm{~kg} / \mathrm{dia}$ \\
\hline Ácido acético & $\begin{array}{l}5.88^{*} 10^{\wedge}-3 \\
\text { ton/dia }\end{array}$ & $5.88 \mathrm{~kg} / \mathrm{dia}$ \\
\hline Agua & $\begin{array}{l}0.092 \\
\text { ton/dia }\end{array}$ & $92 \mathrm{~kg} / \mathrm{dia}$ \\
\hline
\end{tabular}

En la tabla 7 se muestran las cantidades de producción de manzanas en unidades de toneladas por año, y la cantidad de ácido acético (vinagre) producidos, así como el agua producida toneladas por día.
Finalmente, la tabla 8 muestra las dimensiones para el diseño de un biorreactor que pueda producir 5,88 kilogramos por día de ácido acético. Se observan las condiciones que se necesitan para una producción eficaz de ácido acético a nivel industrial, permitiendo ver que el diseño del reactor es clave para que la producción sea óptima e ideal.

\section{Tabla 7.}

Dimensiones del biorreactor equipado con una turbina de Rushton

\begin{tabular}{|l|l|}
\hline Volumen de etanol & $0.0276 \mathrm{~m}^{3}$ \\
\hline Volumen de agua & $0.078 \mathrm{~m}^{3}$ \\
\hline Volumen total (al $75 \%)$ & $0.105 \mathrm{~m}^{3}$ \\
\hline Volumen total del reactor & $0.140 \mathrm{~m}^{3}$ \\
\hline Diámetro del impelente & $0.14 \mathrm{~m}$ \\
\hline Diámetro del reactor & $0.42 \mathrm{~m}$ \\
\hline
\end{tabular}

Por su parte, la figura 1 muestra un esquema del biorreactor diseñado en la presente investigación.

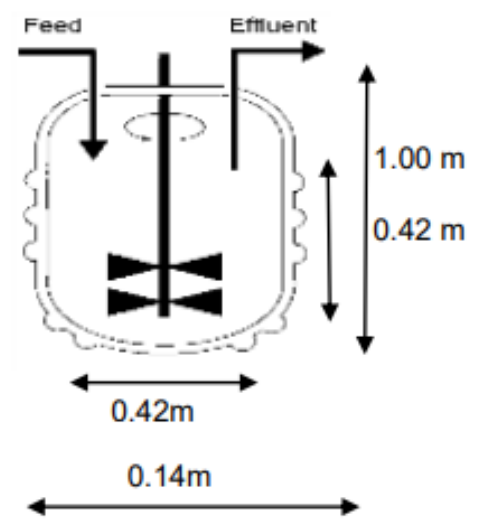

Figura 1. Diseño del biorreactor de producción de ácido acético

\section{Conclusiones}

Las bacterias presentes en la "madre del vinagre" transformaron el $60 \%$ del sustrato alcohólico, las variables 
sustrato, producción de biomasa y producto permite obtener los coeficientes del cultivo celular. Los rendimientos experimentales fueron comparados con los rendimientos máximos termodinámicos de biomasa/sustrato y de producto / sustrato. Los cálculos realizados para el diseño del reactor sugieren que se puede mejor el rendimiento de producción de vinagre a escala industrial ya que si bien es cierto la fermentación se puede lograr sin la agitación, al utilizar la turbina para agitación se logra dispersar de forma óptima el aire en el medio obteniendo así una mayor cantidad de producto y mayores rendimientos productivos.

\section{Agradecimientos}

Los autores expresan su agradecimiento a quienes conforman el grupo del Laboratorio Central de la Universidad Técnica de Manabí.

\section{Bibliografía}

Alawad, S. M., Sulieman, A. M. E., Osman, M. A., \& Mudawi, H. A. (2018). Production and Quality Evaluation of Vinegar from Tamarind (Tamarindus indica L.) Fruit Pulp. Gezira Journal of Engineering and Applied Sciences, 10(1).
Aleixandre, J. L., Padilla, A. I., Navarro, L. L., Suria, A., García, M. J., \& Álvarez, I. (2003). Optimization of making barrel-fermented dry Moscatel wines. J. Agric. Food Chem. 51, 1889-1893.

de Ory, I., Romero, L. E., \& Cantero, D. (2002). Optimum startingup protocol of a pilot plant scale acetifier for vinegar production. Journal of Food Engineering, 52(1), 31-37.

Doran, P. (2013). Bioprocess Engineering Principles. Elsevier, USA.

Drysdale, G. \& Fleet, G. (1988). Acetic Acid Bacteria in WineMaking: A Review. American Journal of Enology and Viticulture, 39, 143-154.

Ferreyra, M. M., Schvab, M. D. C., Davies, C. V., Gerard, L. M., \& Solda, C. A. (2014). Obtención de vinagre de naranja en proceso semicontinuo, a escala laboratorio. Ciencia, docencia y tecnología, 25(49), 154-165.

Geankopolis, J. (1999). Procesos de transporte $y$ operaciones unitarias. CECSA, México.

Heikefelt, C. (2011). Chemical and sensory analyses of juice, cider and vinegar produced from different apple cultivars. Degree Project for MSc Thesis in Horticulture, Horticultural Science Programme, EX0544, SLU, Swedish University of 
Agricultural Sciences, Sweden.

Hurtado, M. A., Ramos, I. M., Parrado, D. S., \& Guzmán, H. E. (s.f.). Aislamiento e Identificación de Bacterias Ácido Acéticas en Materia Prima y Tren de Fermentación en el Ingenio Providencia SA.

Jimenez-Hornero, J.E., SantosDuenas, I., \& García-García, I. (2009). Optimization of biotechnological processes. The acetic acid fermentation. Part I: The proposed model, en: Biochemical Engineering Journal, 45(1): 1-6

Johnston, C., Steplewska, I., Long, C., Harris, L., \& Ryals, R. (2010). Examination of the Antiglycemic Properties of Vinegar in Healthy Adults. Ann Nutr Metab 56: 74-79

Shishehbor, F., Mansoori, A., Sarkaki, A., Jalali, M., \& Latifi,
S. (2008). Apple cider vinegar attenuates lipid profile in normal and diabetic rats. Pak $J$ Biol Sci. Dec 1;11 (23): 2634-2638.

Soler, A., \& Buitrago, G. (2010). Evaluación de la transferencia de oxígeno en cultivos con lactococcus lactis empleando un sistema de fermentación con aireación externa. Revista Colombiana de Biotecnología, 12(2), 124-138.

Solieri, L. (2009). «Storage» de Vinegars of the World, Springer Science \& Business Media. p. 149.

Teneda-Llerena, W. F., \& Milla-Toro, S. O. (2018). Estudio para determinar el mercado potencial del vinagre de frutas naturales: Aplicación en el Ecuador. UDAAKADEM, (2), 88-115. 